\title{
Revascularisation in diabetics with multivessel coronary artery disease
}

\author{
K J Beatt, K P Morgan, A Kapur
}

Heart 2004;90:999-1002. doi: 10.1136/hrt.2003.028852

$\mathrm{D}$ abetic patients are recognised as being at high risk of vascular complications in a variety of situations. Approximately $80 \%$ will die of a cardiovascular event. In recent years there has been increasing recognition of the diversity of mechanisms responsible for prevalence of adverse events, although there are still many aspects that are poorly understood. Coronary artery disease is the major cause of death among diabetics and tends to be more severe and diffuse in this group. The growth of the diabetic population combined with recent technological and pharmacological advances in both bypass surgery and angioplasty make choosing the optimum revascularisation strategy in this group one of the most challenging issues facing the cardiologist today.

The exponential relation between the risk of developing diabetes mellitus (DM) and increasing body mass index ensures that the incidence of type 2 DM will rapidly increase if the current trend in western countries of increasing weight each succeeding generation continues. This is particularly relevant to immigrant communities moving to cultures enjoying a higher standard of living; not only do they have a higher incidence of diabetes, but their growth in population tends to be proportionately greater than the growth in the indigenous population. This statistic suggests that DM will continue to consume an increasing proportion of medical resources, not least the provisions set aside for the treatment of coronary artery disease.

\section{THE DIABETIC PROCESS}

Not only do diabetics have a greater complexity and extent of vascular disease in general, but they also have the additional disadvantages of having multisystem dysfunction involving endothelium, platelets, and renal and neurological systems.

The primary defect in type 2 DM is not fully understood, but the pathophysiology driving the disease process can be divided into four areas: endothelial dysfunction, platelet and clotting abnormalities, lipid abnormalities, and the consequences of hyperglycaemia, including protein and collagen modifications. All four interact with each other to produce a cycle of progression affecting every organ system in the body. The consequence of this pathophysiological process on the coronary arterial vasculature is a tendency towards smaller calibre coronary vessels and a more severe diffuse type of coronary disease.

\section{REVASCULARISATION IN DIABETICS WITH MULTIVESSEL DISEASE: THE STORY SO FAR}

Nearly all of the data comparing percutaneous coronary intervention (PCI) and coronary artery bypass graft surgery $(\mathrm{CABG})$ in diabetic patients with multivessel disease relates to comparisons made in the late 1980s and 1990s. These studies tend to demonstrate increased rates of revascularisation in the PCI group but no difference in either survival, non-fatal myocardial infarction (MI) or cerebrovascular accident (CVA) (table 1$) \cdot{ }^{1-9}$
Given the advances in CABG and especially PCI over the past decade it is difficult to justify extrapolating these data to the present day. Additionally, all of the randomised trials comparing bypass surgery with angioplasty involved highly selected populations and this is particularly true when considering the subset of patients with diabetes. The percentage of patients with DM recruited into randomised trials varies from $6-19 \%$, and the outcomes in sample populations recruited into trials may not accurately reflect the outcome of the general diabetic population.

Particular emphasis has been placed on the results of the BARI (bypass angioplasty revascularisation investigation) trial which completed in 1984 and involved 324 patients with DM. ${ }^{3}$ The five year mortality was $19.4 \%$ among 180 patients assigned to CABG and $34.5 \%$ among 173 patients assigned to PCI $(p<0.003)$. The large difference in mortality between the two arms strongly suggests that between 1988 and 1991 diabetics were better off with surgical revascularisation. ${ }^{6}$

The clinical relevance of this analysis should be examined further. While these patients were not stratified in the randomisation process, their baseline characteristics were similar and crossover between arms was minimal. However, registry data reveals that only $16 \%$ of eligible patients were actually randomised. Furthermore the advantage of surgery over PCI was only seen in those patients who received a left internal mammary artery (LIMA) graft to the left anterior descending (LAD) artery ( $81 \%$ of the diabetics)-in the absence of a LIMA graft, PCI and CABG conferred equal benefit. The PCI arm also experienced a $10 \%$ rate of abrupt closure and $8 \%$ rate of emergency CABG, levels much higher than seen in current clinical practice. In addition, there was no assurance that diabetic control was maintained in the diabetics either in the initial in-hospital phase or during long term follow up, something that may have important implications for the outcome of revascularised diabetic patients. Lastly this trial was conducted in an era that preceded stenting and the adjunctive pharmacotherapy now available. Clinical developments would suggest that the BARI data have little relevance to current clinical practice.

Data from the more recent ARTS (arterial revascularisation therapies study) published in 2001 compared CABG to

Abbreviations: BARI, bypass angioplasty revascularisation investigation; $C A B G$, coronary artery bypass graft surgery; $C A B R I$, coronary angioplasty versus bypass revascularisation; CREDO, clopidogrel for the reduction of events during observation; CVA, cerebrovascular accident; DES, drug eluting stents; DM, diabetes mellitus; EAST, Emory angioplasty versus surgery trial; EPIC, evaluation of 7E3 for the prevention of ischaemic complications; EPILOG, evaluation in PTCA to improve long-term outcome with abciximab GP $\mathrm{Illb} / \mathrm{llla}$ blockade; EPISTENT, evaluation of platelet $\mathrm{llb} / \mathrm{lll}$ a inhibition for stenting; GABI, German angioplasty bypass surgery investigation; LAD, left anterior descending coronary artery; LIMA, left internal mammary artery; MASS, medicine angioplasty or surgery study; MACE, major adverse cardiac events, $\mathrm{Ml}$, myocardial infarction; $\mathrm{PCl}$, percutaneous coronary angioplasty; RITA, randomised intervention of angina; TLR, target lesion revascularisation; TVR, target vessel revascularisation 
Table 1 Summary of trials comparing PCl with CABG

\begin{tabular}{lllll}
\hline Study & $\begin{array}{l}\text { Total number of } \\
\text { patients }\end{array}$ & $\begin{array}{l}\text { Number of } \\
\text { diabetics }\end{array}$ & $\begin{array}{l}\text { Recruitment } \\
\text { period }\end{array}$ & $\begin{array}{l}\text { CABG superior to PCI in } \\
\text { diabetics }\end{array}$ \\
\hline BARI & 1829 & 353 & $1988-1991$ & Yes \\
CABRI & 1054 & 122 & $1988-1992$ & No \\
EAST & 392 & 59 & $1987-1990$ & No \\
RITA & 1011 & 62 & $1988-1991$ & No \\
ERACI & 127 & 13 & $1988-1990$ & No \\
GABI & 359 & 43 & $1896-1991$ & No \\
ARTS & 1205 & 208 & $1996-1997$ & No \\
ERACI II & 450 & 77 & $1996-1998$ & No \\
\hline \multicolumn{5}{l}{ See footnote on first page for explanation of trial acronyms. } \\
\end{tabular}

multivessel PCI and stenting. ${ }^{10}$ In 1205 patients randomised there was no difference in the composite end point of death, MI or CVA ( $8.7 \%$ CABG $v 9.4 \%$ PCI arm, $\mathrm{p}=\mathrm{ns}$ ) at one year; however, there were more repeat revascularisation procedures in the PCI arm (3.5\% CABG $v 16.8 \%$ PCI, p < 0.001). There were 208 diabetics included in the 1205 patients randomised. This group again was not stratified prospectively, but the diabetic patients in each arm (112 in the PCI, 96 in the CABG arm) had similar baseline characteristics. The rate of the combined end point of death/CVA/MI was similar in the two arms ( $12.5 \%$ CABG $v 17 \%$ PCI, $p=n s)$. The requirement for additional revascularisation was higher after PCI (3.1\% CABG $v 25 \%$ PCI, p $<0.01)$, but was reduced in both diabetic and non-diabetic subgroups compared to trials from the pre-stent era. These findings suggest an improvement in periprocedural complications compared to the earlier trials, but little change on the impact of restenosis.

\section{ADVANCES IN PCI TECHNOLOGY}

The benefits of intracoronary stenting are well established with a reduction in restenosis rates and clinical events most pronounced in diabetics. Despite these improvements, the main limitation of PCI in diabetics continues to be the high rate of restenosis. Even in the stent era studies demonstrate DM to be an independent risk factor for restenosis, with restenosis rates following stent implantation in some studies in excess of $50 \%$ depending on lesion length and/or the diameter of the lumen.

\section{PLATELET INHIBITION}

The most significant and earliest development in pharmacological adjunctive treatment for PCI is platelet inhibition. The benefits were proven even in the pre-stent era. Since then the optimum regimen of aspirin and a thienopyridine has been implemented. Clopidogrel is now the thienopyridine of choice following safety concerns associated with the use of ticlopidine. Aspirin and clopidogrel work synergistically together to deliver enhanced inhibition of platelet aggregation.

It is accepted that patients who have undergone PCI should remain on aspirin long term. However, the optimum duration for thienopyridine treatment remains less well characterised. $\mathrm{O}$ 'Neil and colleagues found no difference in outcome with long term thienopyridine treatment post-PCI. ${ }^{11}$ However, subset analysis shows a tendency in the diabetic subgroup towards improved survival, which was non-significant.

More recently the CREDO (clopidogrel for the reduction of events during observation) investigators examined the effect of long term (12 month) administration of clopidogrel postPCI compared to one month. ${ }^{12}$ They found that long term thienopyridine treatment was associated with a $26.9 \%$ relative reduction in risk of the composite end point of death/MI or CVA. Approximately a quarter of patients included in the study were diabetic. Interestingly subgroup analysis of these patients showed the reduction in relative risk was less when compared to non-diabetics although this did not achieve statistical significance.

The EPIC, EPILOG, and EPISTENT trials all examined the efficacy of a periprocedural infusion of glycoprotein IIb/IIIa inhibitor as adjunctive therapy in PCI. The EPILOG (evaluation in PTCA to improve long-term outcome with abciximab GP IIb/IIIa blockade) investigators compared the outcomes of 638 diabetic patients enrolled in the trial to non-diabetic patients. ${ }^{13}$ They found during hospitalisation a composite of death, MI or urgent revascularisation occurred in $7.1 \%$ of diabetics compared to $7.5 \%$ of non-diabetics. At six months the composite risk of death or MI was $8.8 \%$ for diabetics and $7.4 \%$ for non-diabetics. Treatment with the glycoprotein IIb/ IIIa inhibitor abciximab significantly reduced the composite end point of death or MI among both groups ( 0.28 and 0.47 at 30 days and 0.36 and 0.60 at six months for diabetics and non-diabetics, respectively).

In the more recent EPISTENT (evaluation of platelet IIb/ IIIa inhibition for stenting) trial the benefit of stenting and adjunctive glycoprotein IIb/IIIa treatment was especially pronounced in the diabetic subgroup. ${ }^{14}$ Diabetics receiving abciximab had a $48 \%$ relative reduction in composite event rate of death, MI or target vessel revascularisation (TVR) ( $25 \%$ v 13\%) with the benefit extending to one year follow up. Interestingly major adverse cardiac event (MACE) rates in the stent plus abciximab group for diabetics and nondiabetics were similar (18.6\% $v$ 20.5\%) suggesting this combination of treatment abolished the excess risk associated with PCI in diabetics.

A pooled analysis of the three abciximab trials showed a reduction in one year mortality in the diabetic subgroup from $4.5 \%$ to $2.5 \%(\mathrm{p}=0.031$ ) compared to $2.6 \%$ to $1.9 \%$ in nondiabetics $(\mathrm{p}=0.1) \cdot{ }^{15}$

\section{DRUG ELUTING STENTS IN DIABETICS}

Specific data for the diabetic subsets have been reported in the RAVEL (randomised study with the sirolimus-eluting velocity balloon-expandable stent) and the SIRIUS (sirolimuseluting stent in de novo native coronary lesions) studies, using the Cypher sirolimus coated stent, and in Taxus IV using the Taxus paclitaxel coated stent.

In the RAVEL trial where there were relatively few diabetics recruited $(\mathrm{n}=44)$, there was a dramatic difference in the primary end point of late luminal loss at six months $(0.08 \mathrm{~mm}$ for the sirolimus group against $0.82 \mathrm{~mm}$ for the bare metal stent group, $\mathrm{p}<0.0001) .{ }^{16}$ This is a sensitive measurement of post-PTCA intimal proliferation and probably provides the best index of the extent of restenosis.

Data from the SIRIUS investigators support the encouraging results from RAVEL. In the SIRIUS trial, which included a more complex patient population consisting of $26 \%$ diabetics $(\mathrm{n}=279)$, small vessels and complex lesions (type $\mathrm{B} 2$ and $\mathrm{C}=58.6 \%$ ), the investigators reported consistent reductions in late loss and restenosis in the diabetic subgroup. ${ }^{17}$ At nine months $12.2 \%$ of diabetics treated with 
drug eluting stents (DES) reached the primary end point of target vessel failure (a composite of cardiac death, MI, or target vessel revascularisation) compared to $27.0 \%$ of patients receiving bare metal stents. The diabetic patient subset treated with DES showed an absolute reduction in insegment restenosis from $50.5 \%$ to $17.6 \%$. This treatment effect was large compared to the non-diabetic group, where the absolute reduction was smaller; from $31.2 \%$ to $6.1 \%$. In this trial target lesion revascularisation (TLR) was driven by symptoms and not by the angiographic findings. At one year TLR rates were dramatically reduced in the DES group $(20.0 \%$ $v 4.9 \%$ ). In the diabetic subgroup the TLR rate was $27.1 \%$ in the control group and $8.8 \%$ in the Cypher group.

Diabetics with small vessels $(\leqslant 2.5 \mathrm{~mm})$ and longer lesions $(\geqslant 15 \mathrm{~mm}$ ) demonstrated an unprecedented $43.1 \%$ $(66.8 \% \vee 23.7 \%)$ absolute reduction of restenosis rates with $64.5 \%$ relative reduction. Patients at lower risk, like nondiabetics, with large vessels ( $\geqslant 3 \mathrm{~mm}$ in diameter) and short lesions ( $\leqslant 12 \mathrm{~mm}$ in length) presented a more modest $15.2 \%$ $(18.6 \%$ v 3.4\%) absolute reduction of restenosis, which still represents a high relative reduction of $81.7 \%$.

In the more recently reported Taxus IV study comparing the paclitaxel eluting Taxus stent to an identical uncoated stent a broadly similar population were recruited: a total of 1326 patients with a quarter of patients being diabetic and $57 \%$ of patients received glycoprotein IIb/IIIa inhibitors.

At nine month follow up there was no difference between the two groups with regard to the incidence of death or MI. However, TLR and TVR rates, as well as MACE and target vessel failure rates, were significantly lower in the Taxus arm of the study.

In the diabetic subgroup, the Taxus stent was associated with a $68 \%$ relative risk reduction in TLR rates compared to bare metal stenting ( $16.0 \% \vee 5.2 \%$, respectively; $p<0.001)$. This is almost identical to the $69 \%$ relative reduction in TLR seen in the diabetic subgroup receiving DES in the SIRIUS study $(22.9 \% \quad v \quad 7.2 \%)$. Although both studies recruited primarily patients from North America the absolute difference in TLR rates may indicate important and unidentified differences in the recruited populations. Nevertheless the threefold reduction in the TLR rate in the diabetic group compared to the non-diabetic group is impressive and makes a strong case for the routine use of coated stents in diabetics.

\section{CURRENT STATUS}

While there is growing evidence for the use of DES and platelet inhibitors in diabetics undergoing PCI, it is less clear how diabetic patients should be selected for PCI.

With recognised advances in the treatment of diabetics with PCI the question now arises: have these improvements reached the stage when PCI can challenge surgery as the optimal revascularisation strategy in multivessel diabetics?

To answer this question the CARDia (coronary artery revascularisation in diabetes) trial has been set up in the UK and Ireland. It is an investigator initiated study and is the first prospective study designed specifically to address the hypothesis: optimal PCI with stenting and abciximab is not inferior to up-to-date CABG as a revascularisation strategy for diabetics with multi-vessel or complex single vessel coronary disease (fig 1). The primary end point is the well established composite of death, non-fatal MI, or CVA at one year. Twenty one centres in the UK are a third of the way through recruiting 600 diabetic patients randomised to PCI or surgery, and a further group randomised to a bare metal versus Cypher stents. Recruitment is due to be completed in 2004

It remains to be seen if DES, coupled with the use of adjunctive therapy such as glycoprotein IIb/IIIa inhibitors and optimal diabetic control, will establish PCI as the

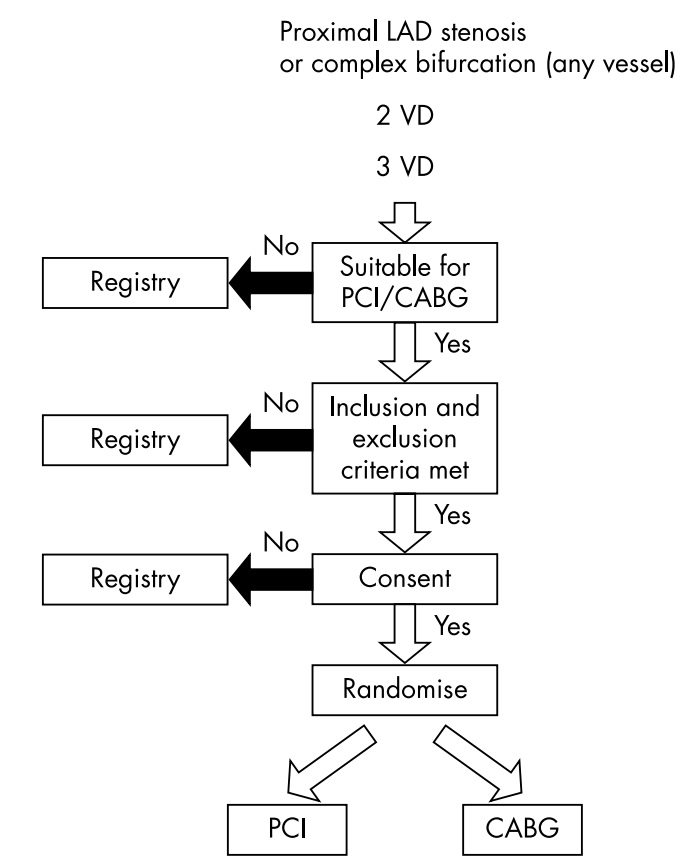

Primary end point:
Occurrence of death/non-fatal MI/ stroke at 1 year

Figure 1 Flow diagram of inclusion criteria for the CARDia trial. $C A B G$, coronary artery bypass graft surgery; $L A D$, left anterior descending coronary artery; $\mathrm{PCl}$, percutaneous coronary intervention.

treatment of choice for diabetics. The emerging data suggest that treatment with DES appears to convey added benefit to the diabetic patient and is the stent of choice when undertaking PCI.

\section{Authors' affiliations}

K J Beatt, K P Morgan, A Kapur, Hammersmith Hospitals NHS Trust, London, UK

Correspondence to: Dr Kevin J Beatt, Hammersmith Hospitals NHS Trust, Du Cane Road London, UK; k.beatt@imperial.ac.uk

\section{REFERENCES}

1 RITA Investigators. Coronary angioplasty versus coronary artery bypass surgery. The randomized intervention treatment of angina (RITA) trial. Lancet 1993:341:573-80.

2 CABRI Trial Participants. First-year results of CABRI (coronary angioplasty versus bypass revascularisation investigation). Lancet 1995;346:1179-84

3 BARI Investigators. Comparison of coronary bypass surgery with angioplasty in patients with multivessel disease. The bypass angioplasty revascularization investigation (BARI) investigators. N Engl J Med 1996:335:217-25.

4 Carrie D, Elbaz M, Puel J, et al. Five-year outcome after coronary angioplasty versus bypass surgery in multivessel coronary artery disease: results from the French monocentric study. Circulation 1997:96:11-6.

5 Goy JJ, Eeckhout E, Burnand B, et al. Coronary angioplasty versus left internal mammary artery grafting for isolated proximal left anterior descending artery stenosis. Lancet 1994;343:1449-53.

6 Hamm CW, Reimers J, Ischinger T, et al. A randomized study of coronary angioplasty compared with bypass surgery in patients with symptomatic multivessel coronary disease. German angioplasty bypass surgery investigation (GABI). N Engl J Med 1994;331:1037-43.

7 Hueb WA, Bellotti G, de Oliveira SA, et al. The medicine, angioplasty or surgery study (MASS): a prospective, randomized trial of medical therapy, balloon angioplasty or bypass surgery for single proximal left anterior descending artery stenoses. J Am Coll Cardiol 1995:26:1600-5.

8 King SB III, Lembo NJ, Weintraub WS, et al. A randomized trial comparing coronary angioplasty with coronary bypass surgery. Emory angioplasty versus surgery trial (EAST). N Engl J Med 1994;331:1044-50.

9 Rodriguez A, Boullon F, Perez-Balino N, et al. Argentine randomized trial of percutaneous transluminal coronary angioplasty versus coronary artery bypass surgery in multivessel disease (ERACI): in-hospital results and 1-year follow-up. ERACl group. J Am Coll Cardiol 1993;22:1060-7. 
10 Serruys PW, Unger F, Sousa JE, et al. Comparison of coronary-artery bypass surgery and stenting for the treatment of multivessel disease. N Engl J Med 2001;344:1117-24

11 O'Neill WW, Serruys P, Knudtson M, et al. Long-term treatment with a platelet glycoprotein-receptor antagonist after percutaneous coronary revascularization. EXCITE trial investigators. evaluation of oral xemilofiban in controlling thrombotic events. N Engl J Med 2000;342:1316-24

12 Steinhubl SR, Berger PB, Mann JT III, et al. Early and sustained dual oral antiplatelet therapy following percutaneous coronary intervention: a randomized controlled trial. JAMA 2002:288:2411-20.

13 Kleiman NS, Lincoff AM, Kereiakes DJ, et al. Diabetes mellitus, glycoprotein $\mathrm{llb} / \mathrm{lll}$ a blockade, and heparin: evidence for a complex interaction in a multicenter trial. EPILOG investigators. Circulation 1998;97:1912-20.
14 Marso SP, Lincoff AM, Ellis SG, et al. Optimizing the percutaneous interventional outcomes for patients with diabetes mellitus: results of the EPISTENT (evaluation of platelet Ilb/llla inhibitor for stenting trial) diabetic substudy. Circulation 1999;100:2477-84.

15 Bhatt DL, Marso SP, Lincoff AM, et al. Abciximab reduces mortality in diabetics following percutaneous coronary intervention. J Am Coll Cardiol 2000;35:922-8.

16 Morice MC, Serruys PW, Sousa JE, et al. A randomized comparison of a sirolimus-eluting stent with a standard stent for coronary revascularization. N Engl J Med 2002;346:1773-80.

17 Moses JW, Leon MB, Popma JJ, et al. Sirolimus-eluting stents versus standard stents in patients with stenosis in a native coronary artery. N Engl J Med 2003;349:1315-23.

\section{IMAGES IN CARDIOLOGY}

\section{Silent right ventricular myocardial infarction: the $Q$ wave never lies}

A 78 year old patient was referred for a cardiology opinion following the incidental discovery of Q waves in leads III and $\mathrm{aVF}$ on a 12 lead ECG (upper panel). There was also ST segment depression in leads I, aVL, V5, and V6 and electrical evidence of left ventricular hypertrophy. There was no definite history suggestive of myocardial ischaemia and cardiovascular risk factors included previous smoking and hypertension. On examination the patient's blood pressure was 182/88 $\mathrm{mm} \mathrm{Hg}$.

Transthoracic echocardiography showed preserved left ventricular (LV) and right ventricular (RV) systolic function with normal dimensions. In particular there was no inferior wall motion abnormality.

In order to resolve this conflict in information cardiac magnetic resonance imaging (CMR) was undertaken (Siemens Sonata 1.5T system with a phased array chest coil). LV function and dimensions were normal and no wall motion abnormality was present. However, the RV was hypokinetic with an ejection fraction of $43 \%$. Delayed hyperenhancement imaging for myocardial infarction was performed 10 minutes postintravenous contrast injection $(0.1 \mathrm{mmol} / \mathrm{kg}$ gadolinium DTPA). This revealed an extensive transmural RV myocardial infarction as indicated by the arrows (lower panel).

CMR confirmed the diagnosis of isolated $\mathrm{RV}$ infarction and demonstrates that this may lead to chronic RV systolic impairment. Symptomatic isolated RV infarction is uncommon and the prevalence of silent RV ischaemia is unknown. Confirmation of the diagnosis of myocardial infarction is always of clinical importance and secondary prevention has now been advised with aspirin, a $\beta$ blocker, an angiotensin converting enzyme inhibitor, and a statin.

T N Martin

H Dargie t.martin@clinmed.gla.ac.uk
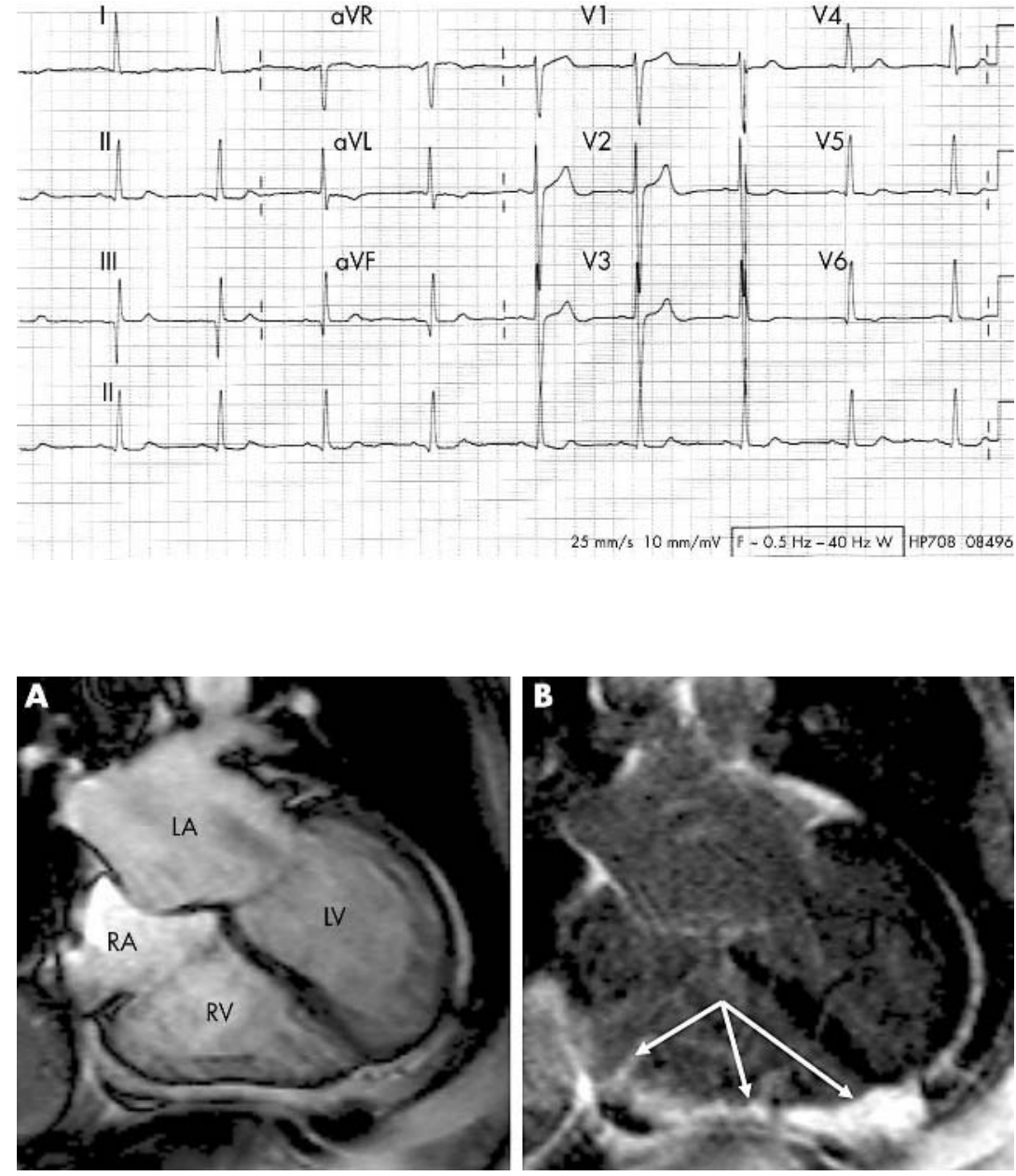

Four chamber views. (A) Diastolic frame from trueFISP CINE images. (B) Delayed enhancement image. The arrows indicate the region of myocardial infarction in the right ventricle. LA, left atrium $\mathrm{LV}$, left ventricle; RA, right atrium; RV, right ventricle. 